\title{
Insulin-like growth factor-binding protein 2 and 5 are differentially regulated in ovarian cancer of different histologic types
}

\author{
Huamin Wang ${ }^{1}$, Daniel G Rosen ${ }^{1}$, Hua Wang ${ }^{2}$, Gregory N Fuller ${ }^{1}$, Wei Zhang ${ }^{1}$ \\ and Jinsong Liu ${ }^{1}$ \\ ${ }^{1}$ Department of Pathology, The University of Texas MD Anderson Cancer Center, Houston, TX, USA and \\ ${ }^{2}$ Department of Gastrointestinal Medical Oncology, The University of Texas MD Anderson Cancer Center, \\ Houston, TX, USA
}

\begin{abstract}
The family of insulin-like growth factor-binding proteins (IGFBPs) comprises six members, which bind and regulate the functions of insulin-like growth factors. Overexpression of IGFBP2 and IGFBP5 contributes to the invasiveness and progression of several human cancers, but their role and clinical significance in ovarian cancer has not been investigated in detail. We examined IGFBP2 and IGFBP5 expression levels using two tissue microarrays, one containing six normal surface epithelium, six benign serous cysts, 10 serous borderline tumors, eight low-grade, and 20 high-grade serous carcinomas. The other comprising 441 ovarian cancers of different histologic types linked to a clinicopathologic database. Each tumor was sampled in duplicate with a 1.0-mm punch core needle. Immunohistochemical staining was performed using antibodies against IGFBP2 or IGFBP5. The staining intensity was scored semiquantitatively as negative (0), weak (1-10\%), moderate (10$50 \%$ ), or strong (50-100\%) using computerized image analysis. Statistical analyses used Fisher's exact test and Kaplan-Meier method. IGFBP2 and IGFBP5 were overexpressed in high-grade serous carcinomas compared to normal surface epithelium, benign serous cysts, serous borderline tumors, or low-grade serous carcinoma. They were differentially expressed in different types of ovarian carcinomas, being more often expressed at high levels in high-grade serous carcinoma, malignant mixed mullerian tumors and undifferentiated carcinoma, and more often expressed at low levels or not at all in clear cell and mucinous carcinomas. We concluded that IGFBP2 and IGFBP5 might play a role in the development of high-grade ovarian serous carcinoma, but not in mucinous or clear cell ovarian carcinomas.
\end{abstract}

Modern Pathology (2006) 19, 1149-1156. doi:10.1038/modpathol.3800637; published online 26 May 2006

Keywords: IGFBP2; IGFBP5; ovarian carcinoma

The insulin-like growth factors (IGFs) play a key role in the tumorigenesis of human malignancies mainly through their antiapoptotic activities or by enhancing tumor cell proliferation. ${ }^{1,2}$ The mitogenic and antiapoptotic activities of IGFs are regulated by six IGF-binding proteins (IGFBPs), namely IGFBP1 to IGFBP6. IGFBPs either inhibit or enhance the action of IGFs, depending on cell type and physiologic/ pathologic status, resulting in either suppression or stimulation of cell proliferation. ${ }^{1,2}$ Among the six IGFBPs, IGFBP2 is overexpressed in many malignancies such as prostatic cancer, human glioma,

Correspondence: Dr J Liu, MD, PhD, Department of Pathology, Unit 085, The University of Texas MD Anderson Cancer Center, 1515 Holcombe Blvd., Houston, TX 77030, USA.

E-mail: jliu@mdanderson.org

Received 6 March 2006; revised and accepted 26 April 2006; published online 26 May 2006 etc. $^{3}$ Overexpression of IGFBP2 in these tumors is often associated with increased aggressiveness of the tumor. ${ }^{4,5}$ For example, increased IGFBP2 expression after castration promotes the growth of androgen-independent human prostate cancer. ${ }^{5}$ IGFBP2 has also been reported to be overexpressed only in glioblastomas, but not in low-grade gliomas and is associated with tumor progression and poor survival in patients with diffuse gliomas. ${ }^{6,7}$ A recent study has shown that overexpression of IGFBP2 may contribute to the invasiveness of glioblastoma by enhancing MMP-2 gene expression. ${ }^{4}$

Ovarian cancer cells express not only IGFs and IGF-1 receptor, but also several isoforms of IGFBPs. ${ }^{8,9}$ Among the IGFBPs expressed in ovarian cancers, IGFBP2 is markedly increased and significantly higher in cystic fluid from malignant epithelial ovarian tumors than in cystic fluid from benign epithelial ovarian tumors, mainly due to the 
increase in mRNA and protein expression in the tumor cells. ${ }^{10}$ Serum IGFBP2 levels are also significantly higher in patients with ovarian cancer compared with benign controls. ${ }^{11,12}$ Serum IGFBP2 levels in patients with ovarian cancers correlates positively with the ovarian tumor marker, CA125. ${ }^{11,12}$ Further study showed that serum IGFBP2 level correlated with tumor stage, grade and survival in patients with ovarian cancer. ${ }^{12}$ Treatment of OVCAR3 human epithelial ovarian cancer cells with IGFBP2 stimulated cell proliferation and activated multiple members of mitogen-activated protein kinase pathways, suggesting that IGFBP2 has an autocrine effect on ovarian cancer cell proliferation. ${ }^{13}$ Analyses of gene expression profiles using cDNA microarrays have shown that IGFBP2 is overexpressed in the cisplatin-resistant ovarian cancer cell lines, KFr and KFrP200, compared to cisplatin-sensitive $\mathrm{KF}$ ovarian cancer cells. ${ }^{14} \mathrm{~A}$ more recent study from our laboratory showed that overexpression of IGFBP2 enhanced the invasiveness of ovarian cancer cells. ${ }^{15}$ SKOV3 ovarian cancer cells stably transfected to overexpress IGFBP2 have 1.8to 2.9-folds higher invasive capacities compared with the parental or vector-transfected SKOV3 cells. ${ }^{15}$ These findings strongly suggest that IGFBP2 plays a role in the tumorigenesis and aggressiveness of ovarian cancer. However, the clinical significance of IGFBP2 expression in ovarian cancer tissue samples has not been investigated in detail.

IGFBP5 is also expressed in ovarian cancers. ${ }^{9}$ However, its role in tumorigenesis, if any, is not clear at this time. It has been reported that IGFBP5 promoted the growth of prostate cancer cells in vitro and accelerated the progression to androgen independence in prostate cancer animal models. ${ }^{16,17}$ Serum IGFBP5 levels in ovarian cancer patients are significantly higher at relapse than at initial diagnosis, suggesting that IGFBP5 is involved in the progression of ovarian cancer. ${ }^{12,18}$ To examine the possible role of IGFBP2 and IGFBP5 in ovarian cancer, we evaluated their expression in 441 ovarian carcinomas of different histologic types using tissue microarray and immunohistochemistry. We also examined the expression of IGFBP2 and IGFBP5 in another tissue microarray containing samples from normal ovaries, benign serous cystadenomas, serous borderline ovarian tumors, low-grade, and highgrade serous carcinomas. The immmunohistochemical staining results were correlated with clinicopathologic parameters including tumor grades, histologic types, stages, and patients' survival.

\section{Materials and methods}

\section{Patient Samples}

Samples used in this study were from patients with primary epithelial ovarian cancers who had undergone initial surgery at The University of Texas MD Anderson Cancer Center between 1990 and 2001. A total of 441 such patients were identified for whom tissue samples and follow-up information were available. Fellow-up was updated through June 2003 by reviewing medical records and the US Social Security Index. In addition, six cases of normal ovarian surface epithelium, six benign serous cysts, 10 serous borderline tumors, eight low-grade serous carcinomas, and 20 high-grade serous carcinomas were included in a separate tissue microarray block. The use of all archival paraffin-embedded tissue blocks and chart reviews were approved by the institutional review board of MD Anderson Cancer Center.

Demographic and survival data for each specimen were entered into a comprehensive database created with Microsoft Access (version 97). Histopathologic diagnoses were based on World Health Organization criteria. Serous carcinomas were graded according to a two-tier (low-grade and high-grade) system proposed by Malpica et $a l^{19}$ For statistical analysis grade 2 and grade 3 endometrioid ovarian carcinomas were grouped as 'high grade' and grade 1 tumors as 'low grade'. Disease stage was assigned according to the International Federation of Gynecology and Obstetrics (FIGO) system. ${ }^{20-24}$ Diseasespecific survival rates were calculated as the percentage of patients who survived with disease for a defined period, reported as time since diagnosis or treatment, and only deaths from the disease were counted.

\section{Construction of Tissue Microarrays}

To construct the two tissue microarrays used in this study, formalin-fixed, paraffin-embedded archival tissue blocks and their matching hematoxylin and eosin-stained slides were retrieved, reviewed and screened for representative tumor regions by a pathologist. The tissue microarrays were constructed with a tissue microarrayer (Beecher Instruments, Sun Prairie, WI, USA) as described previously. ${ }^{25}$ We created two tissue microarrays sets. The first one consisting of 441 ovarian carcinoma cases and the second one consisting of six cases of normal ovarian surface epithelium, six benign serous cysts, 10 serous borderline tumors, eight low-grade serous carcinomas, and 20 high-grade serous carcinomas. The composition of tumors in the tissue microarrays is listed in Table 1. Each tumor or normal ovarian controls were sampled in duplicate from representative areas of either one or two donor blocks using a 1.0-mm punch. The two tissue cores from each tumor were then incorporated into two duplicate tissue microarrays blocks.

\section{Immunohistochemical Staining}

Immunohistochemical analysis was performed using polyclonal antibodies against IGFBP2 and IGFBP5 (Santa Cruz Biotechnology Inc., Santa Cruz, 
Table 1 Expression of IGFBP2 and IGFBP5

\begin{tabular}{|c|c|c|c|c|c|c|}
\hline & \multicolumn{3}{|c|}{ IGFBP2 (\%) } & \multicolumn{3}{|c|}{ IGFBP5 (\%) } \\
\hline & $0+1$ & $2+3$ & $\mathrm{P}$-value & $0+1$ & $2+3$ & P-value \\
\hline \multicolumn{7}{|l|}{ Tumor type } \\
\hline Serous carcinoma & $41(19 \%)$ & $173(81 \%)$ & a & $88(40 \%)$ & $130(60 \%)$ & a \\
\hline Endometrioid carcinoma & $10(28 \%)$ & $26(72 \%)$ & 0.33 & $21(60 \%)$ & $14(40 \%)$ & 0.04 \\
\hline Mixed type carcinoma & $31(32 \%)$ & $66(68 \%)$ & 0.01 & $38(41 \%)$ & $54(59 \%)$ & 0.97 \\
\hline Transitional carcinoma & $3(43 \%)$ & $4(57 \%)$ & 0.28 & $4(57 \%)$ & $3(43 \%)$ & 0.61 \\
\hline Mucinous adenocarcinoma & $5(63 \%)$ & $3(37 \%)$ & 0.01 & $6(86 \%)$ & $1(14 \%)$ & 0.05 \\
\hline Clear cell carcinoma & $14(82 \%)$ & $3(18 \%)$ & $<0.0001$ & $14(82 \%)$ & $3(18 \%)$ & 0.001 \\
\hline MMMT & $3(17 \%)$ & $15(83 \%)$ & 0.95 & $6(33 \%)$ & $12(67 \%)$ & 0.73 \\
\hline Undifferentiated carcinoma & $2(18 \%)$ & $9(82 \%)$ & 0.75 & $3(27 \%)$ & $8(73 \%)$ & 0.58 \\
\hline \multicolumn{7}{|l|}{ Tumor grade } \\
\hline Low grade & $8(50 \%)$ & $8(50 \%)$ & $0.06^{\mathrm{b}}$ & $10(62 \%)$ & $6(38 \%)$ & $0.22^{\mathrm{b}}$ \\
\hline High grade & $100(26 \%)$ & $289(74 \%)$ & & $170(44 \%)$ & $219(56 \%)$ & \\
\hline \multicolumn{7}{|l|}{ Disease stage } \\
\hline Stage I & $12(37 \%)$ & $20(63 \%)$ & $0.15^{\mathrm{c}}$ & $16(52 \%)$ & $15(48 \%)$ & $0.31^{\mathrm{c}}$ \\
\hline Stage II & $8(30 \%)$ & $19(70 \%)$ & $0.71^{\mathrm{d}}$ & $15(58 \%)$ & $11(42 \%)$ & $0.84^{\mathrm{d}}$ \\
\hline Stage III & $73(27 \%)$ & $197(73 \%)$ & $0.29^{\mathrm{e}}$ & $111(41 \%)$ & $157(59 \%)$ & $0.37^{\mathrm{e}}$ \\
\hline Stage IV & $13(18 \%)$ & $61(82 \%)$ & $0.12^{\mathrm{f}}$ & $36(46 \%)$ & $42(54 \%)$ & $0.53^{\mathrm{f}}$ \\
\hline Unstaged & $2(100 \%)$ & $0(0 \%)$ & $0.10^{\mathrm{g}}$ & $2(100 \%)$ & $0(0 \%)$ & $0.19^{g}$ \\
\hline
\end{tabular}

MMMT: malignant mixed mullerian tumor.

${ }^{\mathrm{a}}$ Each histotype compared to serous carcinoma.

${ }^{\mathrm{b}}$ Low vs high grade.

${ }^{\mathrm{C}}$ Overall stage.

${ }^{\mathrm{d}}$ Stage I vs II.

${ }^{\mathrm{e}}$ Stage I vs III.

${ }^{\mathrm{f}}$ Stage III vs IV.

${ }^{g}$ Early stage vs advanced stage.

CA, USA). These type-specific antibodies do not cross-react with the other isoforms of IGFBPs. A standard indirect immunoperoxidase procedure (ABC-Elite; Vector Laboratories, Burlingame, CA, USA) was used for all stains. In brief, antigen retrieval was performed by treatment of the unstained slides in a steamer for $25 \mathrm{~min}$. Antibodies against IGFBP2 or IGFBP5 (both diluted 1:500) were added to tissue sections of the ovarian microarrays and incubated at $4{ }^{\circ} \mathrm{C}$ overnight, followed by incubation with secondary antibody at room temperature for $60 \mathrm{~min}$. Mayer's hematoxylin staining was used as a counterstain. IGFBP2 and IGFBP5 immunohistochemical staining was scored semiquantitatively using Metavue, 6.1 (Molecular Device Corp., Sunnyvale, CA, USA), a computerized image analysis system. Cytoplasmic immunostaining for IGFBP2 and IGFBP5 was measured as the total integrated optical density and expressed in arbitrary optic density units. For statistical analysis, all cases displaying total integrated optical density (mean \pm s.e.) where then group together in a 1-3 scale. Negative was defined as the total absence of cytoplasmic staining and given a score of ' 0 '. For statistical analysis cases exhibiting negative and weak expression (score 1) were grouped as 'Low' expression. Intermediate or strong expression (score 2 and 3) was also grouped together as 'High'. The average score from the two tissue cores of each tumor was used as the final score for IGFBP2 and IGFBP5 staining.

\section{Statistical Analysis}

The statistical analyses were performed using Fisher's exact test, the Spearman correlation and the Kaplan-Meier method. A $P$-value $<0.05$ was considered statistically significant.

\section{Results}

Representative micrographs of immunohistochemical stains for IGFBP2 and IGFBP5 in normal ovarian surface epithelium, benign serous cyst, serous borderline tumor, and high-grade serous carcinoma are shown in Figure 1. Normal ovarian surface epithelium (Figure 1a), benign ovarian serous cyst (Figure 1b), serous borderline tumor (Figure 1c and d) had no or low expression of IGFBP2 or IGFBP5. In contrast, high-grade serous carcinomas showed diffuse strong cytoplasmic staining for both IGFBP2 and IGFBP5 (Figure 1e and f). IGFBP2 was increasingly overexpressed in normal ovarian surface epithelium, serous cystadenomas, serous tumors of low-malignant potential (LMP) low-grade and 

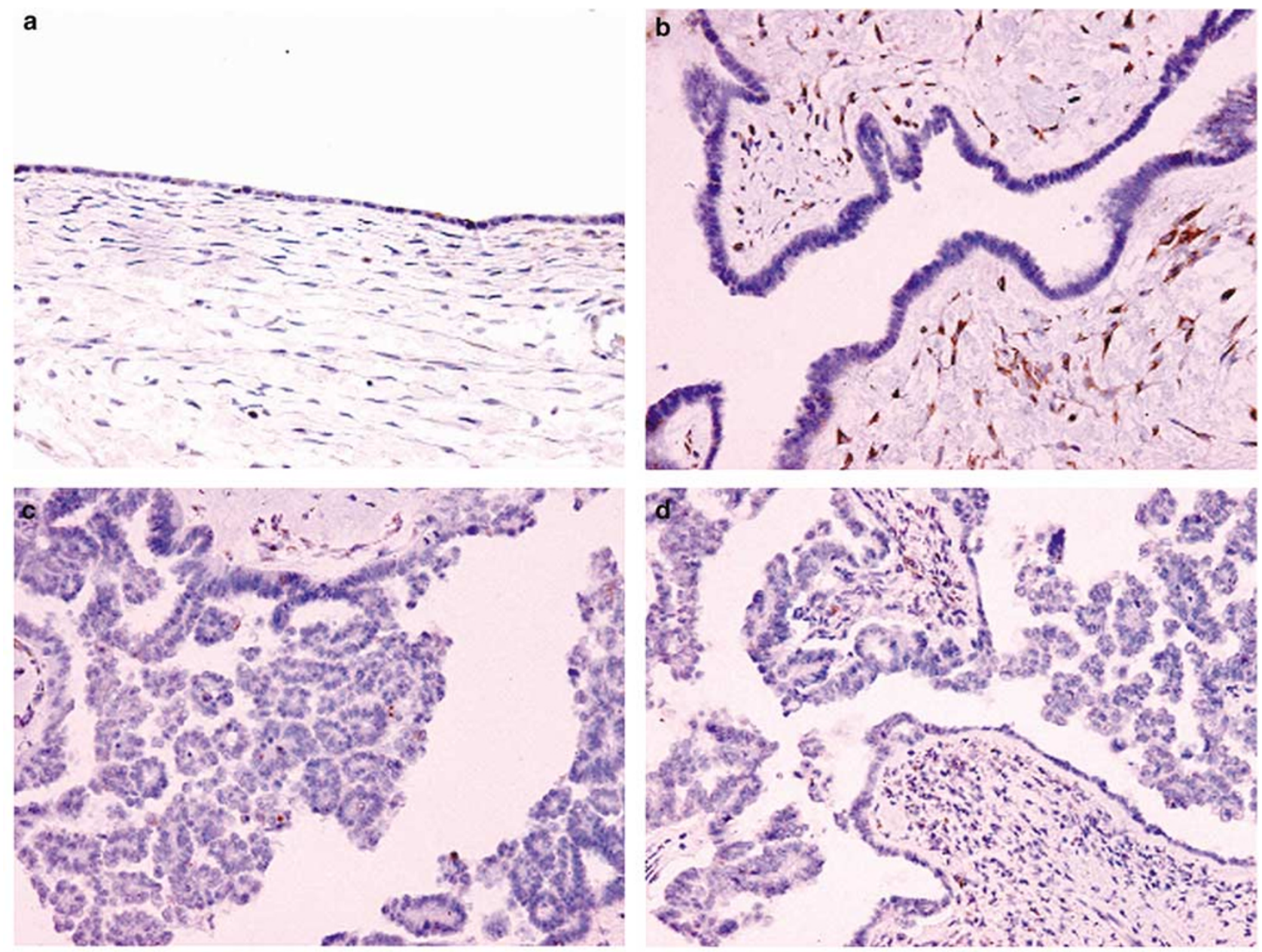

\section{vit}
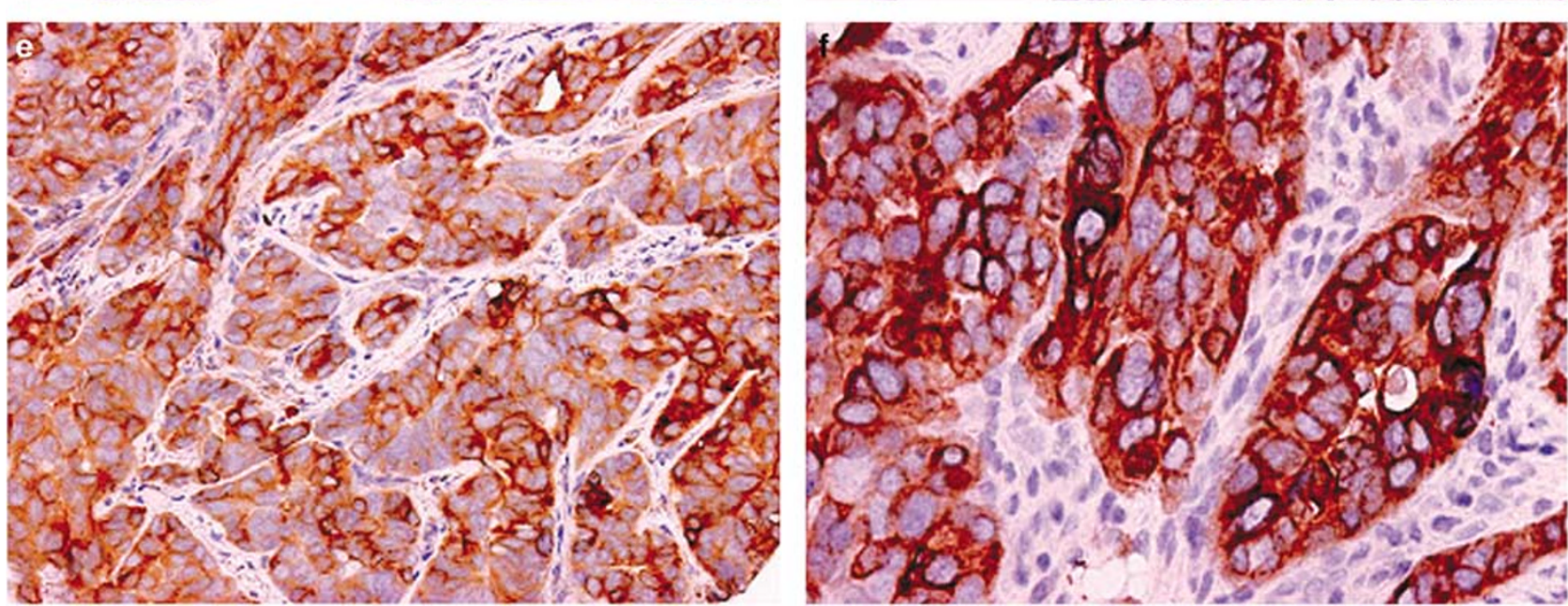

Figure 1 Representative micrographs showing IGFBP2 and IGFBP5 expression. (a) Normal ovarian surface epithelium negative for IGFBP2. (b) Benign serous cystadenoma negative for IGFBP5. Serous borderline tumor, negative for (c) IGFBP2 and (d) IGFBP5 (a-d, original magnification $\times 100$ ). High-grade serous carcinoma strongly positive for (e) IGFBP2 and (f) IGFBP5 (original magnification $\times 200)$.

high-grade serous carcinomas (Figure 2a). Weak or moderate staining for IGFBP2 (a score of 1 or 2) was detected in one of six normal ovarian surface epithelium (17\%), six of six benign serous cysts, seven of 10 serous borderline tumors and six of eight low-grade serous carcinomas $(75 \%)$. Compared to the normal ovarian surface epithelium, the expression of IGFBP2 was significantly higher in benign serous cysts, serous borderline tumors and lowgrade serous carcinomas $(P<0.05)$. However, none of these tumors showed strong (a score of 3) cytoplasmic staining for IGFBP2. No significant 


\section{a IGFBP2 score}

\begin{tabular}{|c|c|c|c|c|c|}
\hline & 0 & 1 & 2 & 3 & p value \\
\hline Normal ovary & 00000 & $\nabla$ & & & \\
\hline Serous cyst & & 0000 & $\infty$ & & 0.008 \\
\hline Serous LMP & $\Delta \infty \Delta$ & 00000 & & & 0.09 \\
\hline Low grade & 00 & 00000 & 0 & & 0.05 \\
\hline High grade & & $\Delta 0$ & 00000 & $8888^{\circ}$ & $<0.000$ \\
\hline
\end{tabular}

\section{b IGFBP5 score}

\begin{tabular}{r|ccccc} 
Normal ovary & 0 & 1 & 2 & 3 & $\mathrm{p}$ value \\
Serous cyst & 00000 & & & & \\
Serous LMP & 0000 & & & & NS \\
0000 & $\diamond$ & & & 0.7 \\
Low grade & 00000 & $\diamond$ & $\diamond$ & & 0.5 \\
High grade & $\diamond$ & $\diamond$ & 000088888 & 0.0003
\end{tabular}

Figure 2 Expression of IGFBP2 (a) and IGFBP5 (b) in tissue microarray consisting of normal ovarian surface epithelium, benign serous cysts, serous borderline tumors, low-grade and high-grade serous carcinomas. The expression levels of IGFBP2 and IGFBP5 were expressed as 0 (negative), 1 (weakly positive), 2 (moderately positive) or 3 (strongly positive). Each diamond represents one case. The $P$-values compared to normal ovarian surface epithelium are shown on the right. NS, no significance.

difference was found in IGFBP2 expression between low-grade serous carcinomas and benign serous cysts or serous borderline tumors (Figure 2a). In contrast, 13 of 20 high-grade serous carcinomas $(65 \%)$ showed strong cytoplasmic staining for IGFBP2. High-grade serous carcinomas had significant higher levels of IGFBP2 compared to low-grade serous carcinomas, serous borderline tumors, benign cysts or normal ovarian surface epithelium $(P<0.01$, Figure 2a). IGFBP5 expression was also significantly higher in high-grade serous carcinoma than in lowgrade serous carcinomas, serous borderline tumors, benign cysts or normal ovarian surface epithelium $(P<0.01$, Figure 2b). However, IGFBP5 expression was similar in normal ovarian surface epithelium, benign serous cysts, serous borderline tumors and low-grade serous carcinomas $(P>0.05$, Figure $2 b)$. These results suggested that IGFBP2 and IGFBP5 might play a role in the development of high-grade serous carcinoma. The differences in IGFBP2 and IGFBP5 expression between high-grade and lowgrade serous carcinomas also suggest that different molecular mechanisms are involved in the development of these two types of tumors.

To further examine IGFBP2 and IGFBP5 expression in ovarian carcinomas of different histologic types and to determine their prognostic significance, we examined the expression of both markers using tissue microarrays consisting of 441 ovarian carcinomas. Moderate (score 2) to strong (score 3) IGFBP2 expression was detected in 83,82 , and $81 \%$ of malignant mixed mullerian tumor (MMMT), undifferentiated carcinoma and serous carcinomas, respectively, which were significantly higher compared to mucinous $(P=0.01)$ and clear cell carcinomas $(P<0.0001)$ (Table 1). Endometrioid and mixed type carcinomas also expressed higher levels of IGFBP2 than did clear cell carcinomas but similar proportions to serous histotype. Representative stains for IGFBP2 in the various tumor types are shown in Figure 3. Similar to IGFBP2, moderate to strong expression of IGFBP5 was detected in 67, 73, 60 , and 59\% in MMMT, undifferentiated carcinoma, serous carcinomas, and mixed carcinomas compared to $14 \%$ in mucinous carcinoma $(P=0.05)$ and $18 \%$ in clear cell carcinoma $(P=0.001)$ (Table 1). In 16 of the 18 MMMT, both spindle cell and epithelial component was sampled. We could not observe differences between spindle cell and epithelial component of MMMT tumors (data not shown). Representative stains for IGFBP5 in the various tumor types are shown in Figure 4. These results suggest that IGFBP2 and IGFBP5 are differentially regulated in different types of ovarian carcinomas.

In terms of tumor grade, 74 and $56 \%$ of high-grade tumors showed moderate to strong staining for IGFBP2 and IGFBP5, respectively, compared to 50 and $38 \%$ in low-grade carcinomas. Survival analysis showed that serous carcinomas with weak or no IGFBP2 expression had a trend to better long-term survival than tumors with moderate to strong IGFBP2 expression, but the difference was not statistically significant $(P=0.09$, Figure 5$)$. No correlation between IGFBP2 expression and survival time was observed in the overall study group (data not shown). With regard to IGFBP5, no correlation was found with survival in either the overall study group or the serous carcinoma group $(P>0.05$, data not shown).

Finally, the expression of IGFBP2 correlated with that of IGFBP5 in ovarian carcinoma group (Figure $6)$. Only $6 \%$ of ovarian carcinomas with low IGFBP2 expression ( 0 or 1 ) had moderate to strong expression of IGFBP5. In contrast, $76 \%$ of ovarian carcinomas with high levels of IGFBP2 (2 or 3) also showed moderate to strong expression of IGFBP5 $(P<0.0001)$. However, expression levels of IGFBP2 were higher than IGFBP5 in serous carcinomas and endometrioid carcinomas $(P<0.001)$.

\section{Discussion}

In this study, we found that both IGFBP2 and IGFBP5 were overexpressed in high-grade serous carcinomas compared to normal surface epithelium, benign serous cysts, serous borderline tumors, and low-grade serous carcinoma. Moreover, IGFBP2 and 

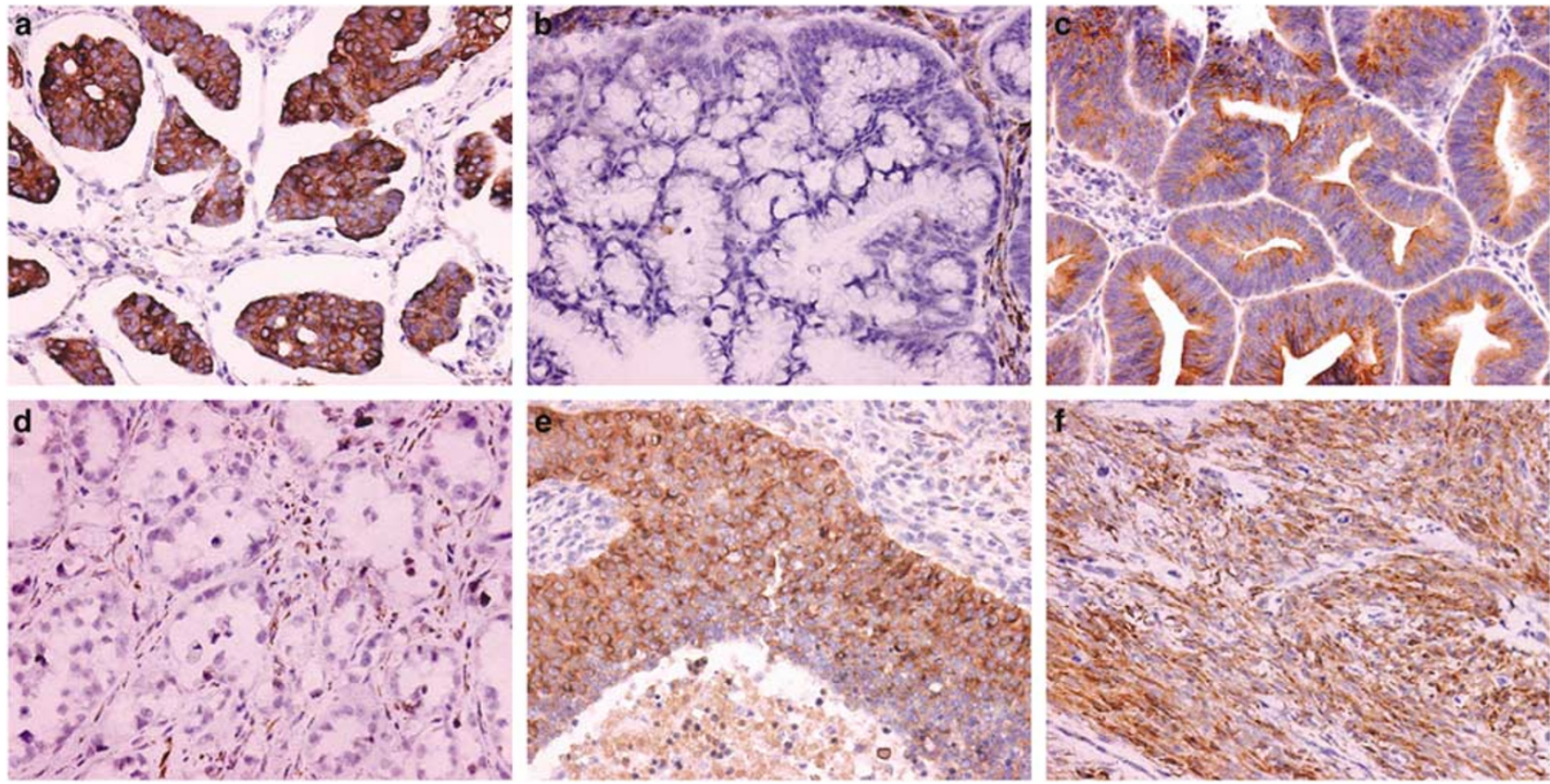

Figure 3 Representative tissue cores showing the expression of IGFBP2 in (a) serous carcinoma, (b) mucinous carcinoma, (c) Endometrioid carcinoma, (d) clear cell carcinoma, (e) undifferentiated carcinoma, and (f) malignant mixed mullerian tumor (original magnification $\times 200$ ).
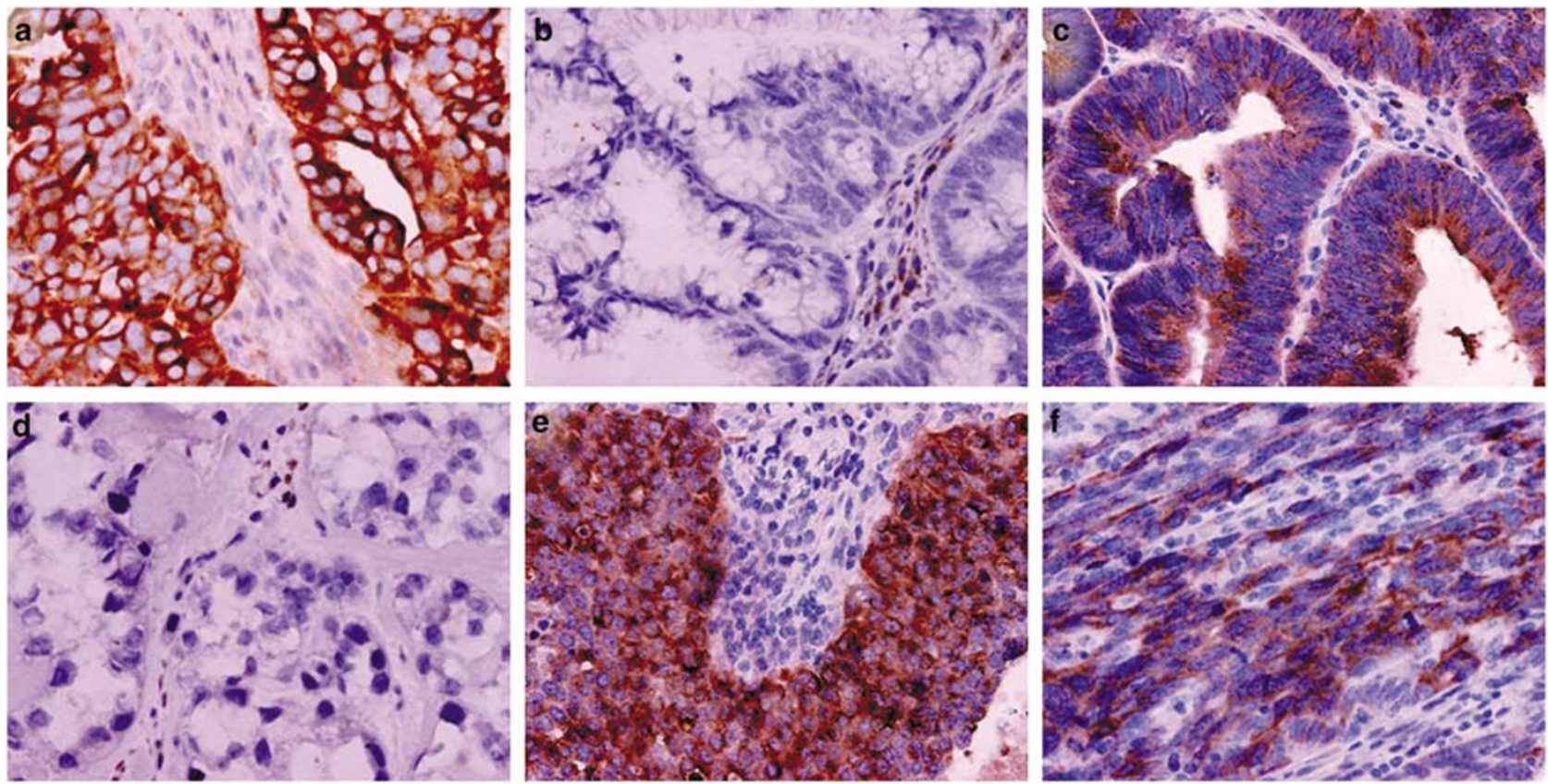

Figure 4 Representative tissue cores showing the expression of IGFBP5 in (a) serous carcinoma, (b) mucinous carcinoma, (c) Endometrioid carcinoma, (d) clear cell carcinoma, (e) undifferentiated carcinoma, and (f) malignant mixed mullerian tumor (original magnification $\times 200$ ).

IGFBP5 were differentially expressed in different histologic types of ovarian carcinomas, being expressed at high levels in high-grade serous carcinoma, MMMT, and undifferentiated carcinoma, but low or no expression in clear cell or mucinous carcinoma. Therefore, our data support the notion that both IGFBP2 and IGFBP5 play an important role in the development of high-grade serous cancers, but not clear cell or mucinous carcinomas.

The degree of heterogeneity and the expression pattern of the marker under study may constitute a potential problem in tissue microarray-based studies, particularly in MMMT and other high-grade carcinomas. The representativity of tissue micro- 

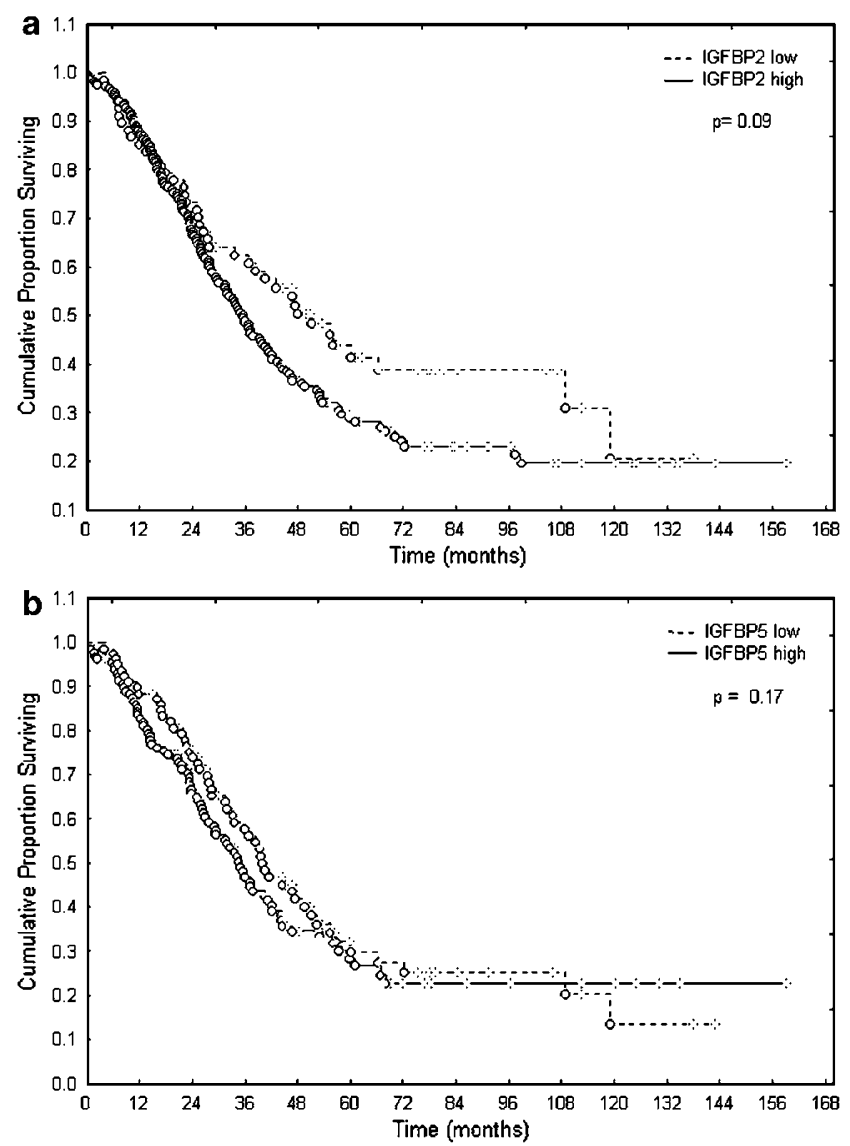

Figure 5 Kaplan-Meier survival curves of patients with serous carcinomas. The expression of (a) IGFBP2 and (b) IGFBP5 was categorized as low (staining score of 0 or 1 ) or high (staining score of 2 or 3 ).

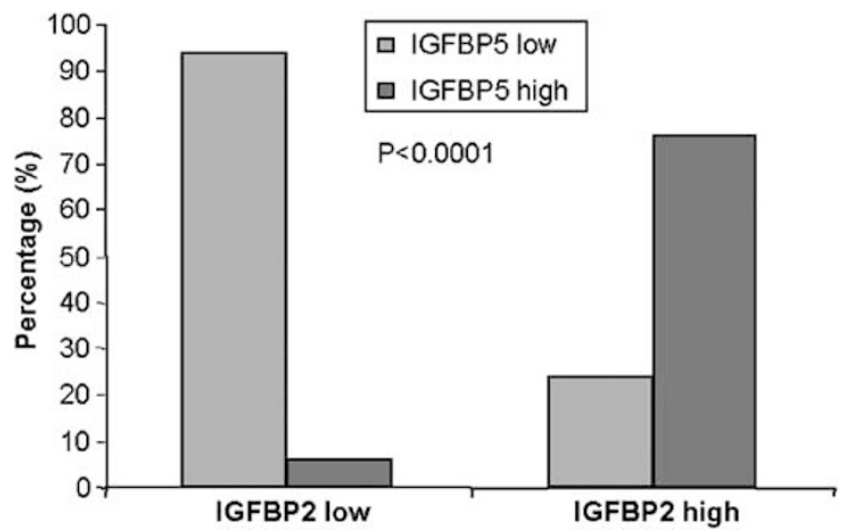

Figure 6 Expression of IGFBP2 correlates with the expression of IGFBP5 in ovarian carcinomas. The expression of IGFBP2 and IGFBP5 was categorized as low (staining score of 0 or 1 ) or high (staining score of 2 or 3 ).

array-based studies, however, has been carefully examined, and the data published so far have clearly indicated that the frequencies of molecular alterations identified by high-throughput tissue microarray screening analysis generally correspond well with those identified using full-size tissue sections. ${ }^{6,26}$ The antibodies used in this study are type specific. There is no cross-reactivity between antiIGFBP2 antibody and IGFBP5 protein. ${ }^{15}$ We performed Western blot experiments using purified recombinant IGFBP2 and IGFBP5 proteins to determine the specificity of the antibodies. Our data shows that there was no cross-reactivity between anti-IGFBP2 antibody and IGFBP5 protein or between anti-IGFBP5 antibody and IGFBP2 protein (unpublished data). Our immunohistochemical staining results showed that IGFBP2 was differentially overexpressed in different histologic types of ovarian carcinomas with the frequency of expression ranging from 18 to $83 \%$. More than $80 \%$ of the MMMTs, undifferentiated carcinoma and serous carcinoma showed moderate to high levels of IGFBP2 expression in the cytoplasm of tumor cells. These findings are consistent with a previous observation that increases in serum IGFBP2 are caused mainly by the markedly increased production of IGFBP2 by tumor cells in vivo. ${ }^{10}$ In addition, some studies have shown that patients with malignant ovarian tumors have significantly higher serum levels of IGFBP2 compared with benign controls. ${ }^{11,12}$ In contrast, only $18 \%$ of clear cell carcinomas and $37 \%$ of mucinous ovarian carcinomas showed moderate to strong staining for IGFBP2. In the light of our previous results that overexpression of IGFBP2 enhanced the invasiveness of ovarian cancer cells, ${ }^{15}$ findings from this study provide further evidence that IGFBP2 is involved in the development of undifferentiated carcinoma, MMMT, and serous carcinoma, but not of clear cell or mucinous ovarian carcinomas. Consistent with this conclusion is our finding that IGFBP2 expression is progressively increased in normal ovarian surface epithelium to benign serous cysts, serous borderline tumors and low-grade serous carcinomas to high-grade serous carcinomas. Suggesting that IGFBP2 might play a role in tumor aggressiveness. It was particularly interesting that the expression levels of IGFBP2 and IGFBP5 were significantly higher in high-grade serous carcinoma than in lowgrade serous carcinoma, suggesting that low-grade and high-grade serous carcinoma may develop through different molecular pathways. These findings are consistent with those from Lee et al. ${ }^{15}$

Although IGFBP2 expression correlated weakly with tumor grades when clear cell carcinomas were excluded, no correlations were seen between IGFBP2 expression and tumor grade, tumor stage or survival in the overall study group. However, patients with serous carcinoma that expressed low levels of IGFBP2 (0 or 1) appeared to have better long-term survival than those with serous carcinomas that expressed moderate to high levels of IGFBP2. This observation is consistent with previous reports that the serum IGFBP2 levels correlate positively with decreased survival and serum CA-125 levels in ovarian cancer patients. ${ }^{11,12}$

The role of IGFBP5 in relation to malignancy is not clear at this time. It has been reported that serum 
IGFBP5 levels are significantly higher at relapse than at initial diagnosis for patients with ovarian cancer. ${ }^{12,18}$ Using immunohistochemical staining with a type-specific polyclonal antibody against IGFBP5, we found that IGFBP5 was overexpressed in 18-73\% of ovarian carcinomas. Similar to the findings for IGFBP2, MMMT, undifferentiated carcinoma, serous carcinoma and transitional cell carcinomas had significant higher expression levels of IGFBP5 compared to clear cell or mucinous carcinomas. These findings, in combination with the observed correlations between IGFBP5 expression and tumor histology, tumor grade and stage provide strong circumstantial evidence that IGFBP2 and IGFBP5 play a role in ovarian cancer development.

In summary, the results from this study support the hypothesis that IGFBP2 and IGFBP5 are implicated in the development of ovarian carcinoma. The observed differential expression of these two proteins between low-grade and high-grade serous carcinomas and among different histologic types of ovarian carcinoma provides further evidence that different molecular mechanisms are involved in these tumors. Further studies are needed to investigate if inhibition of IGFBP2 and IGFBP5 expression may offer a treatment strategy for targeted ovarian cancer therapy.

\section{References}

1 Macaulay VM. Insulin-like growth factors and cancer. Br J Cancer 1992;65:311-320.

2 LeRoith D, Roberts Jr CT. The insulin-like growth factor system and cancer. Cancer Lett 2003;195:127-137.

3 Hoeflich A, Reisinger R, Lahm H, et al. Insulin-like growth factor-binding protein 2 in tumorigenesis: protector or promoter? Cancer Res 2001;61:8601-8610.

4 Wang $H$, Shen W, Huang H, et al. Insulin-like growth factor binding protein 2 enhances glioblastoma invasion by activating invasion-enhancing genes. Cancer Res 2003;63:4315-4321.

5 Kiyama S, Morrison K, Zellweger T, et al. Castrationinduced increases in insulin-like growth factor-binding protein 2 promotes proliferation of androgenindependent human prostate LNCaP tumors. Cancer Res 2003;63:3575-3584.

6 Wang H, Zhang W, Fuller GN. Tissue microarrays: applications in neuropathology research, diagnosis, and education. Brain Pathol 2002;12:95-107.

7 Fuller GN, Rhee $\mathrm{CH}$, Hess KR, et al. Reactivation of insulin-like growth factor binding protein 2 expression in glioblastoma multiforme: a revelation by parallel gene expression profiling. Cancer Res 1999;59: 4228-4232.

8 Yee D, Morales FR, Hamilton TC, et al. Expression of insulin-like growth factor I, its binding proteins, and its receptor in ovarian cancer. Cancer Res 1991;51: 5107-5112.

9 Conover CA, Hartmann LC, Bradley S, et al. Biological characterization of human epithelial ovarian carcinoma cells in primary culture: the insulin-like growth factor system. Exp Cell Res 1998;238:439-449.
10 Kanety H, Kattan M, Goldberg I, et al. Increased insulin-like growth factor binding protein-2 (IGFBP2) gene expression and protein production lead to high IGFBP-2 content in malignant ovarian cyst fluid. Br J Cancer 1996;73:1069-1073.

11 Flyvbjerg A, Mogensen O, Mogensen B, et al. Elevated serum insulin-like growth factor-binding protein 2 (IGFBP-2) and decreased IGFBP-3 in epithelial ovarian cancer: correlation with cancer antigen 125 and tumorassociated trypsin inhibitor. J Clin Endocrinol Metab 1997;82:2308-2313.

12 Baron-Hay S, Boyle F, Ferrier A, et al. Elevated serum insulin-like growth factor binding protein-2 as a prognostic marker in patients with ovarian cancer. Clin Cancer Res 2004;10:1796-1806.

13 Chakrabarty S, Kondratick L. Insulin-like growth factor binding protein-2 stimulates proliferation and activates multiple cascades of the mitogen-activated protein kinase pathways in NIH-OVCAR3 human epithelial ovarian cancer cells. Cancer Biol Ther 2006;5:189-197.

14 Sakamoto M, Kondo A, Kawasaki K, et al. Analysis of gene expression profiles associated with cisplatin resistance in human ovarian cancer cell lines and tissues using cDNA microarray. Hum Cell 2001;14:305-315.

15 Lee EJ, Mircean C, Shmulevich I, et al. Insulin-like growth factor binding protein 2 promotes ovarian cancer cell invasion. Mol Cancer 2005;4:7.

16 Miyake H, Nelson C, Rennie PS, et al. Overexpression of insulin-like growth factor binding protein-5 helps accelerate progression to androgen-independence in the human prostate LNCaP tumor model through activation of phosphatidylinositol $3^{\prime}$-kinase pathway. Endocrinology 2000;141:2257-2265.

17 Miyake H, Pollak M, Gleave ME. Castration-induced up-regulation of insulin-like growth factor binding protein-5 potentiates insulin-like growth factor-I activity and accelerates progression to androgen independence in prostate cancer models. Cancer Res 2000;60: 3058-3064.

18 Katsaros D, Yu H, Levesque MA, et al. IGFBP-3 in epithelial ovarian carcinoma and its association with clinico-pathological features and patient survival. Eur J Cancer 2001;37:478-485.

19 Malpica A, Deavers MT, Lu K, et al. Grading ovarian serous carcinoma using a two-tier system. Am J Surg Pathol 2004;28:496-504.

20 Scully RE. World Health Organization classification and nomenclature of ovarian cancer. Natl Cancer Inst Monogr 1975;42:5-7.

21 Russell P. The pathological assessment of ovarian neoplasms II: the proliferating 'epithelial' tumours. Pathology 1979;11:251-282.

22 Russell P. The pathological assessment of ovarian neoplasms. III: the malignant 'epithelial' tumours. Pathology 1979;11:493-532.

23 Russell P. The pathological assessment of ovarian neoplasms. I: introduction to the common 'epithelial' tumours and analysis of benign 'epithelial' tumours. Pathology 1979;11:5-26.

24 Shepherd JH. Revised FIGO staging for gynaecological cancer. Br J Obstet Gynaecol 1989;96:889-892.

25 Kononen J, Bubendorf L, Kallioniemi A, et al. Tissue microarrays for high-throughput molecular profiling of tumor specimens. Nat Med 1998;4:844-847.

26 Rosen DG, Huang X, Deavers MT, et al. Validation of tissue microarray technology in ovarian carcinoma. Mod Pathol 2004;17:790-797. 